IDEAS \& OPINION

\title{
DisSOCIATION, CHAOS AND TRANSCENDENT FUNCTION
}

\author{
Petr Bob* \\ Center for Neuropsychiatric Research of Traumatic Stress, Department of Psychiatry and UHSL, First Faculty of \\ Medicine, Charles University, Prague, Czech Republic
}

\begin{abstract}
Psychological findings described in original Janet's formulation of dissociation, Jung's complex theory, Putnam's theory of discrete behavioral states and nonlinear dynamic principles of brain and cognitive processes suggest interesting principles for understanding dissociative processes on levels of competitive neural assemblies and their mental representations. In this context, Jung's term transcendent function that enables integration of dissociated states might be related to nonlinear chaotic processes that could represent its neurophysiological correlate. In these self-organizing systems "linear causality" is replaced by "circular causality" that represents a concept useful for describing multilevel interactions on the level of neurons as well as on the level of consciousness related to archetypal intentional action and images in the mind during process of formatting complexes from the pre-existing archetypes as ordering factors of chaotic fluctuations. From the point of Jungian theory these selforganizing psychophysiological processes may be understood as a source of mental images of psychological wholeness mainly as images of circles and symmetric arrangements that could underly brain functions as well as rules of the human psyche that are connected to experienced union of psychological opposites that integrate dissociated states.
\end{abstract}

Key words: Archetype; Chaos; Complex; Discrete behavioral states; Dissociation; Self-organization; Transcendent function

\section{INTRODUCTION}

Jung's theory of psychological complexes represents interesting concept of dissociative processes originally elaborated by Pierre Janet. In connection with contemporary theory of dissociation recent conceptualizations of archetypes as self-organized patterns of the mind enable theoretical links of psychology with dynamic nonlinear brain processes. This suggests possible relationships between archetypal symbols and dynamic principles of brain functions.

\section{DISSOCIATION AND THE COMPLEX THEORY}

Pierre Janet in his work about psychological automatisms (Janet, 1890) defines dissociation as a deficit of the associated system that creates secondary consciousness, which he called subconscious fixed idea (van der Hart \& Friedman, 1989; Bob, 2003) and similarly Sigmund

*Correspondence to: Petr Bob, e-mail: petrbob@netscape.net

Received July 15, 2013; accepted August 21, 2013; Act Nerv Super (Praha) 55(4), 151-156. 
Freud and Joseph Breuer considered double consciousness in "Studies in hysteria" (Breuer \& Freud, 1895). In contrast to that, Carl Gustav Jung considered dissociation of personality not only as pathological phenomenon (Jung, 1972a), but understood dissociation of psyche as a fundamental psychological process that enables differentiation and specialization of psychic processes, for example, focusing of will or concentration on a single target (Jung, 1972b). During these processes, distinct psychic entities are created and associated with certain contents of memory, patterns of behavior, and emotional charges. Jung called these entities "psychic complexes" and most dominant one of them is "ego-complex". As Janet suggested, fundamental causes of the etiology of pathological complexes are mainly traumatic events, which produce traumatic memories. Complexes thus generate alternate fields of the psyche, and it is possible, by means of these complexes, to explain extreme cases of dissociation such as multiple personality disorder (MPD) (Bob, 2004). In this context, pathological influence of the complex leads to a lowered mental level typical for dissociated states (Janet, 1890; van der Hart \& Friedman, 1989; Bob 2003a).

Jung identified these complexes in his experiments in Burghölzl and described them in his studies of word associations (Jung, 1973). Jung found that when a defect occurs in free associations it is caused by a complex (Jung, 1972c, 1973). These complexes, according to Jung's psychodynamic theory, are created out of inborn and inherent dispositions and their ethological manifestation emerge as patterns of behavior. On psychological level these dispositions act as ordering factors that organize psychic contents, perceptions, and fantasies into complex psychic structures. These psychological ordering factors were described by Jung in his study of psychic regression in schizophrenic patients and also in mythology and dream production, and called them archetypes (Jung, 1968).

From the perspective of the Jung's complex theory, it is very interesting that in hypnosis components of the personality very similar to subpersonalities of the multiple personality were found also in normal individuals (Barret, 1995; Bob, 2004; Bowers \& Brecher, 1955; Lynn et al., 1994; Merskey, 1992; Rickeport, 1992; Watkins, 1993; Watkins \& Watkins, 1979-80). For example, Bowers and Brecher (1995) reported interesting material involved in the emergence of multiple personality structure under hypnosis. The authors concluded that this structure was not produced by the hypnosis, but preceded the beginning of the hypnotic work. The patient in the case under discussion had not shown the multiple structure in clinical and psychological examinations prior to the hypnosis. In his conscious state the patient was not aware of his three underlying personalities, each of which reported distinctive dream material and Rorschach responses. Similarly, Barret (1995) described similarities between states of dreaming and MPD including amnesia and other alterations of memory. This suggests dream characters as hallucinated projections of aspects of the self that can be seen as prototypes for the alter personalities. Extreme early trauma may mutate or overdevelop these dissociated parts, inducing them to function in the external world, and thus leading to development of multiple personality disorder.

\section{DISSOCIATION AND DISCRETE BEHAVIORAL STATES}

Jung's complex theory in its basic concept is similar to theoretical proposal of discrete behavioral states described by Frank Putnam (1997). Discrete behavioral states (DBS) provide alternative perspective for modern understanding of dissociation. The term DBS originates from the study of infant mental states. Infant behavioral states can be defined by a set of observable continuous and dichotomous variables. Healthy children are born with basic set of behavioral states and the number of infant states and their levels of interconnection increase with development. Fundamental features of the system of discrete states of consciousness are different state-dependent behaviors in response to the same stimulus. In adults, this type of differential responsiveness is most apparent in such disorders as bipolar illness or MPD. State defining variables may be continuous or dichotomous and define 
behavioral state space. It means that individual behavioral states exist within larger multidimensional framework or space defined by a chosen set of variables and occupies discrete volumes of state space.

According to this concept an individual's behavior traverses the state space in a series of discontinuous jumps or switches from one state to another. State space may be vast but individual regularly occupies those regions in which one has created stable discrete states. Discrete states as transitory behavioral structures are linked together by directional pathways forming behavioral architecture that defines an individual's personality. Transition between behavioral states is manifested as "switch" that represents abrupt change in the values of the constellation of state defining variables, for example transition from waking to sleeping or in bipolar illness from mania to depression. DBS model defines "pathological dissociation" as a trauma induced discrete behavioral states that are widely separated in multidimensional state space from normal states of consciousness and it corresponds to conventional definition, which emphasize the separation or segregation of specific ideas or affects from normal mental phenomena (Putnam, 1997; Kaplan \& Sadock, 1991). When two types of states are significantly different, then the states are separated by a wide gap in state space that may determine pathological dissociative states. Observable differences between the two discrete states are not a simple function of moving up or down and probably they are linked to nonlinear dynamic features connected to chaos (Putnam, 1997). For example Wolff (1987) highlights differential responsiveness as an example of the nonlinearity of input output relation in different states of consciousness and conceptualizes the relevance of nonlinear dynamic systems theory to discrete behavioral states where switches between behavioral states constitute nonlinear transitions. Further recent studies also suggest that rapid shifts in mood and behavior correspond to nonlinear dynamic processes (Putnam, 1997; Gottschalk et al., 1995).

\section{DISSOCIATION AND CHAOS}

Dissociation represents a special form of consciousness in which events that would ordinarily be connected are divided from one another (Li \& Spiegel, 1992). Dissociative states may be characterized by antagonistic competitive relationship among certain states. These states occur on parallel levels as mental representations of competitive neural assemblies and can be modeled by parallel distributed processing in neural networks (Butler et al., 1996; Li \& Spiegel, 1992; Mc Clelland \& Rumelhart, 1986). Dissociation from the neurophysiological point of view may be explained on levels of the brain complexity and competition among cell assemblies represented as a number of simultaneously active neuronal assemblies, which are involved in performing a task. Competition among cortical neural cell assemblies which excite one another and are unable to agree on a common frequency of oscillations (Freeman, 1993) may be understood as neurophysiological model of dissociated (or disintegrated) mental states (Bob, 2003). When associated strength in these activated ranges of the neural network is low, it leads to strong competition among the cell assemblies (Freeman 1993), which in turn represent these mental representations. In parallel distributed processing these dissociated states with low associated strength are represented by isolated "peaks" corresponding to multi-stable states on mental level as well as in the brain (Butler et al., 1996; Li \& Spiegel, 1992; Mc Clelland \& Rumelhart, 1986).

In some cases of competition among neural assemblies chaotic states may occur that probably represent important aspects of brain dynamics. In the brain, chaos probably arises from the competition of two or more parts of the brain (neuronal assemblies) (Freeman 1991) and on the psychological level their mental representations (Bob, 2003). Chaos often leads to an instantaneous reduction of excitatory thresholds of many neural populations not excited in that particular combination before. On the psychological level, for example, they are observed as unexpected original ideas or in pathological cases for example as epileptic 
paroxysms (Elbert et al., 1994; Freeman, 1991). A characteristic feature of neural activity due to brain chaos is synchronous collective activity - a burst (Freeman, 1991) that is mediated by chaotic competition of neural assemblies corresponding to mental representations of conflicting psychic complexes. Freeman speculates (Freeman, 1991) that chaos underlies the ability of the brain to respond flexibly to the outside world and to generate novel activity patterns, including those that are experienced as fresh ideas. Chaos thus enables to express underlying unpredictable order of attractors and enables the complex behavior of the brain (Freeman, 1991; 2000; 2001; Skarda \& Freeman, 1987).

On the psychological level these neurophysiological processes probably correspond to prototypes of intentional behavior (neurophysiologically located in the limbic system) (Freeman, 2001) and archetypes as ordering factors of conscious and unconscious mental events and behavior, which lead to formation of complexes. These findings suggest that personality may be conceptualized as a non-linear self-organized dynamic system with archetypes as pre-existing principles of organization that within the personality manifest as a psychological complex with impersonal characteristics mediated through myths and rituals or through consciousness (McDowell, 2001; Saunders \& Scar, 2001). These connections suggest possible relationships between cognitive neuroscience and psychodynamic theory of archetypes as primitive conceptual structures (Knox, 2001; von Franz, 1974).

\section{CHAOS AND TRANSCENDENT FUNCTION}

Chaos in the brain per se implicates levels of unpredictability of mental and behavioral events (Freeman, 1999). In this context, discoveries of chaos had profound implications for research of brain functions as dynamical systems related to attractors in the web of synaptic connections modified by prior learning (Skarda \& Freeman, 1987) corresponding to intentional archetypes (Freeman, 2000). These pre-existing chaotic fluctuations (intentional archetypes) are enhanced by input and influence selections of new macroscopic patterns (Freeman, 1999). Typical for these chaotic self-organizing systems is that "linear causality" is replaced by "circular causality" that enables to describe multilevel interactions between microscopic neurons in assemblies and the macroscopic emergent state variables that participate on organization of intentional actions integrating multimodal macroscopic patterns (Freeman, 1999).

Similar principles are manifested also on parallel psychological levels by archetypes of intentional behavior that correspond to images in the mind in the process of formatting complexes from the pre-existing ordering factors. Intentionality thus represents a key concept which enables to link neuron and brain to goal-directed behavior through brain dynamics (Freeman, 2000). According to Freeman an archetypal form of intentional behavior is an act of observation in space-time, by which information is sought for the guidance of future action to explore unpredictable and ever-changing environments. These acts are based in the brain dynamics that creates spatiotemporal patterns of neural activity, serving as images of goals and command sequences (Freeman, 2000).

Circular and chaotic processes on the neurophysiological levels hypothetically might represent psychological events that are linked to multilevel connections of dissociated contents. This psychological archetypal tendency creating connections of conflicting dissociated contents was termed by Jung as "transcendent function" (Jung, 1974) that enables integration of dissociated states and creates novel ideas and insights. In this context, "neurophysiological circularity" and corresponding "psychological circularity" may manifest as mental images reflecting symbols of the Self that usually are represented by circles and symmetric arrangements that might "mirror" underlying brain functions related to union of psychological opposites integrating dissociated states (Jung, 1972). 


\section{CONCLUSION}

Recent findings in neuroscience might provide neurophysiological concept of dissociation on the level of chaotic brain processes and understanding "archetypes" as dynamic ordering factors underlying brain physiology as well as human psyche. These conceptualizations may help to explain basic principles of mind-brain duality and to connect methods of neuroscientific and psychodynamic research.

\section{REFERENCES}

Barret, D. (1995). 'The dream character as prototype for the multiple personality alter'. Dissociation, 8, 66-68.

Bob, P. (2003a). 'Subliminal processes dissociation and the 'I'.' Journal of Analytical Psychology, 48, 307316.

Bob, P. (2003b). 'Dissociation and Neuroscience: History and New Perspectives'. International Journal of Neuroscience, 113, 903-914.

Bob, P. (2004). 'Dissociative processes, multiple personality and dream functions'. American Journal of Psychotherapy, 58(2), 2004 (in press).

Bowers, M. K. \& Brecher, S. (1955). 'The emergence of multiple personalities in the course of hypnotic investigation'. International Journal of Clinical and Experimental Hypnosis, 3, 188-199.

Breuer, J. \& Freud, S. (1895). Studies in hysteria. New York: Basic Books.

Butler, L.D., Duran, R.E.F., Jasiukaitis, P., Koopman, CH. \& Spiegel, D. (1996). 'Hypnotizability and Traumatic Experience: A Diathesis-Stress Model of Dissociative Symptomatology'. American Journal of Psychiatry, Festschrift Supplement, 153, 42-62.

Elbert, T., Ray, W.J., Kowalik, Z.J., Skinner, J.E., Graf, K.E. \& Birbaumer N. (1994). ‘Chaos and Physiology: Deterministic Chaos in Excitable Cell Assemblies'. Physiological Reviews, 74, 1-47.

Freeman, W.J. (1991). 'The Physiology of Perception'. Scientific American, 264, 34-41.

Freeman, W.J. (2000). 'Mesoscopics neurodynamics: From neuron to brain'. Journal of Physiology (Paris), 94, 303-322.

Freeman, W.J. (2001). 'Biocomplexity: Adaptive behavior in complex stochastic dynamical systems'. Biosystems, 59, 109-123.

Gottschalk, A. M., Bauer, M. S. \& Whybrow, P. C. (1995). 'Evidence of chaotic mood variation in bipolar disorder'. Archives of General Psychiatry, 52, 947-959.

Janet, P. (1890). L'Automatisme Psychologique. Paris: Felix Alcan.

Jung, C. G. (1968). Analytical Psychology: Its Theory and Practice. London: Routledge \& Kegan Paul.

Jung, C.G. (1972). 'The transcendent function'. In Collected Works of C. G. Jung. 8. Princeton: University Press (p. 67-91).

Jung, C. G. (1972a). 'On the Nature of the Psyche'. In Collected Works of C.G. Jung 8. Princeton: Princeton University Press.

Jung, C. G. (1972b). 'Psychological factors determining human behaviour'. In Collected Works of C.G. Jung 8. Princeton: Princeton University Press.

Jung, C.G. (1972c). 'A Review of the Complex Theory'. In Collected Works of C.G. Jung 8. Princeton: Princeton University Press.

Jung, C. G. (1973). Collected Works of C. G. Jung 2. Princeton: Princeton University Press.

Kaplan, H. I. \& Sadock, B.J. (1991). Comprehensive Glossary of Psychiatry and Psychology. Baltimore: Williams and Wilkins.

Knox, J. M. (2001). 'Memories, fantasies, archetypes: an exploration of some connections between cognitive science and analytical psychology'. Journal of Analytical Psychology, 46, 613-633.

Li, D. \& Spiegel, D. (1992). 'A Neural Network Model of Dissociative Disorders'. Psychiatric Annals, 22, $144-47$. 
Lynn, S. J., Maré, C., Kvaal, S., Segal, D. \& Sivec, H. (1994). 'The hidden observer, hypnotic dreams, and age regression: Clinical implications'. American Journal of Clinical Hypnosis, 37, 130-142.

Mc Clelland, J.L., Rumelhart, D.E. \& the PDP research group (1986). Parallel Distributed Processing I,II. Cambridge: MIT Press.

McDowell, M. J. (2001). 'The three gorillas: an archetype orders a dynamic system'. Journal of Analytical Psychology, 46, 637-654.

Merskey, H. (1992). 'The manufacture of personalities. The production of multiple personality disorders'. British Journal of Psychiatry, 160, 327-40.

Putnam, F. (1997). Dissociation in Children and adolescents. A developmental Perspective. London, New York: The Guilford Press.

Rickeport, M. M. (1992). 'The interface between multiple personality, spirit mediumship and hypnosis'. American Journal of Clinical Hypnosis, 34, 168-77.

Saunders, P. \& Skar, P. (2001). 'Archetypes, Complexes and Self-Organization'. Journal of Analytical Psychology, 46, 305-323.

Skarda, CH.A., \& Freeman, W.J. (1987). 'How Brains Make Chaos in Order To Make Sense of the World'. Behavioral and Brain Sciences, 10, 161-95.

van der Hart, O. \& Friedman, B., (1989). 'A Reader's Guide to Pierre Janet on Dissociation: A Neglected Intelectual Heritage'. Dissociation, 2, 3-16.

von Franz, M. L. (1974). Number and Time: Reflections Leading toward a Unification of Depth Psychology and Physics. Evanston: Northwestern University Press.

Freeman, W.J. (1999). 'Consciousness, Intentionality, and Causality'. Journal of Consciousness Studies, 6, 143-172.

Watkins, J. G. \& Watkins, H. H. (1979-80). 'Ego states and hidden observers'. Journal of Altered States of Consciousness, 5, 3-18.

Watkins, H. H. (1993). 'Ego-state therapy: an overview'. American Journal of Clinical Hypnosis, 35, 232-40.

Wolff, P. H. (1987). The development of behavioral and emotional states in infancy. Chicago: University Chicago Press. 